\title{
Compiler Transformation of Pointers to Explicit Array Accesses in DSP Applications
}

\author{
Björn Franke and Michael O’Boyle \\ Institute for Computing Systems Architecture (ICSA) \\ Division of Informatics \\ University of Edinburgh
}

\begin{abstract}
Efficient implementation of DSP applications is critical for embedded systems. However, current applications written in C, make extensive use of pointer arithmetic making compiler analysis and optimisation difficult. This paper presents a method for conversion of a restricted class of pointer-based memory accesses typically found in DSP codes into array accesses with explicit index functions. C programs with pointer accesses to array elements, data independent pointer arithmetic and structured loops can be converted into semantically equivalent representations with explicit array accesses. This technique has been applied to several DSPstone benchmarks on three different processors where initial results show that this technique can give on average a $11.95 \%$ reduction in execution time after transforming pointer-based array accesses into explicit array accesses.
\end{abstract}

\section{Introduction}

Embedded processors now account for the vast majority of shipped processors due to the exponential demand in commodity products ranging from cell-phones to power-control systems. Such processors are typically responsible for running digital signal processing (DSP) applications where performance is critical. This demand for performance has led to the development of specialised architectures hand-coded in assembly. More recently as the cost of developing an embedded system becomes dominated by algorithm and software development, there has been a move towards the use of high level programming languages, in particular $\mathrm{C}$, and optimising compilers. As in other areas of computing, programming in $\mathrm{C}$ is much less time consuming than hand-coded assembler but this comes at a price of a less efficient implementation due to the inability for current compiler technology to match hand-coded implementations.

To balance the requirement of absolute performance against program development time, there has been a move to tuning $\mathrm{C}$ programs at the source level. Although these tuned programs may perform well with the contemporary compiler technology for irregular DSP architectures [6], such program tuning frequently makes matters worse for optimising compilers for modern DSPs with large, homogeneous register sets and regular architectures. In particular, DSP 


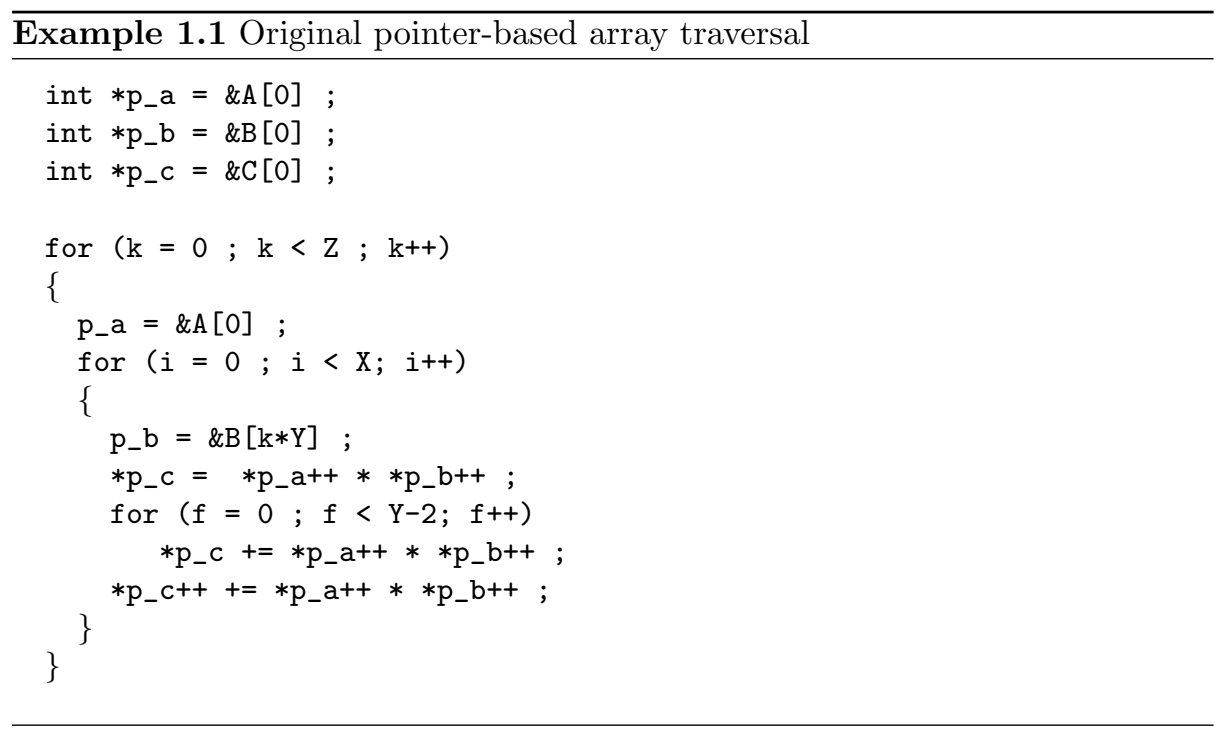

applications make extensive use of pointer arithmetic as can be seen in the DSPstone Benchmarks [13. Furthermore in 10] programmers are actively encouraged to use pointer based code in the mistaken belief that the compiler will generate better code.

This paper is concerned with changing pointer based programs typically found in DSP applications into an array based form amenable to current compiler analysis. In a sense we are reverse engineering "dusty desk" DSP applications for modern high-performance DSPs.

In the next section we provide a motivating example using a typical DSP program and how our technique may transform it into a more efficient form. Section 3 describes the general algorithm and is followed in section 4 by an evaluation of our technique on a set of benchmarks from the DSPstone suite across three platforms. Section 5 describes related work in this area and is followed in section 6 by some concluding remarks.

\section{Motivation}

Pointer accesses to array data frequently occur in typical DSP programs that are tuned for DSPs with heterogeneous register sets and irregular data-paths. Many DSP architectures have specialised Address Generation Units (AGUs) [5], but early compilers were unable to generate efficient code for them, especially in programs containing explicit array references. Programmers, therefore, used pointerbased accesses and pointer arithmetic within their programs in order to give "hints" to the early compiler on how and when to use post-increment/decrement addressing modes available in AGUs. For instance, consider example 1.1 a kernel loop of the DSPstone benchmark matrix2.c. Here the pointer increment 


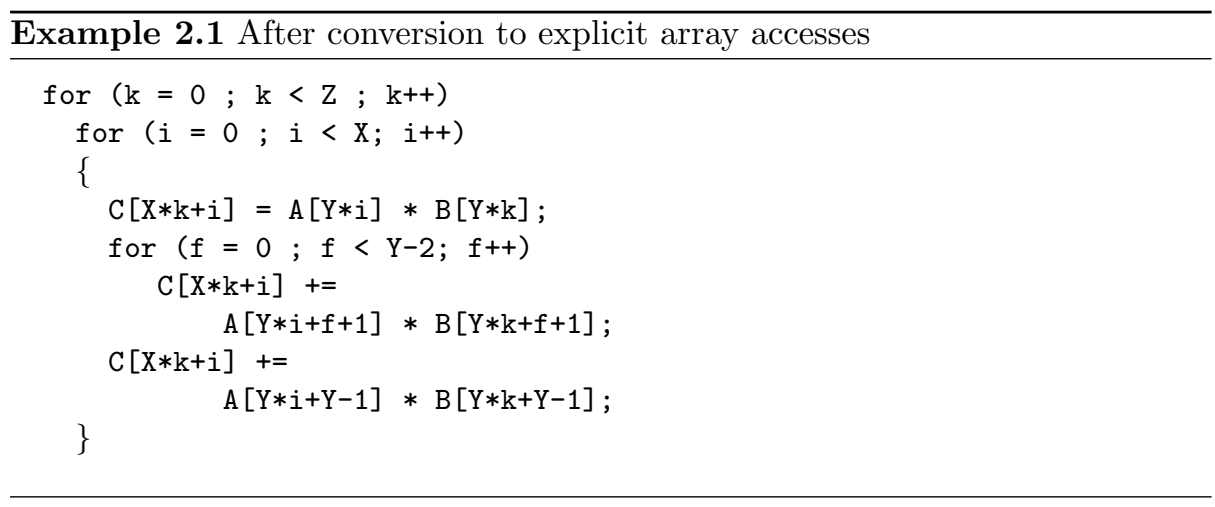

accesses "encourage" the compiler to utilise the post-increment address modes of the AGU of a DSP.

If, however, further analysis and optimisation is needed before code generation, then such a formulation is problematic as such techniques often rely on explicit array index representations and cannot cope with pointer references. In order to maintain semantic correctness compilers use conservative optimisation strategies, i.e. many possible array access optimisations are not applied in the presence of pointers. Obviously, this limits the maximal performance of the produced code. It is highly desirable to overcome this drawback, without adversely affecting AGU utilisation.

Although general array access and pointer analysis are without further restrictions equally intractable [8, it is easier to find suitable restrictions of the array data dependence problem while keeping the resulting algorithm applicable to real-world programs. Furthermore, as array-based analysis is more mature than pointer-based analysis within available commercial compilers, programs containing arrays rather than pointers are more likely to be efficiently implemented. This paper develops a technique to collect information from pointerbased code in order to regenerate the original accesses with explicit indexes that are suitable for further analyses. Furthermore, this translation has been shown not to affect the performance of the AGU [2,5].

Example 2.1 shows the loop with explicit array indexes that is semantically equivalent to example 1.1. Not only it is easier to read and understand for a human reader, but it is amendable to existing array data-flow analyses, e.g. [3. The data-flow information collected by these analyses can be used for e.g. redundant load/store eliminations, software-pipelining and loop parallelisation [14.

A further step towards regaining a high-level representation that can be analysed by existing formal methods is the application of de-linearisation methods. De-linearisation is the transformation of one-dimensional arrays and their accesses into other shapes, in particular, into multi-dimensional arrays [11. The example 2.2 shows the example loop after application of clean-up conversion and de-linearisation. The arrays A, B and C are no longer linear arrays, but have been 
transformed into matrices. Such a representation enables more aggressive compiler optimisations such as data layout optimisations [11. Later phases in the compiler can easily linearise the arrays for the automatic generation of efficient memory accesses.

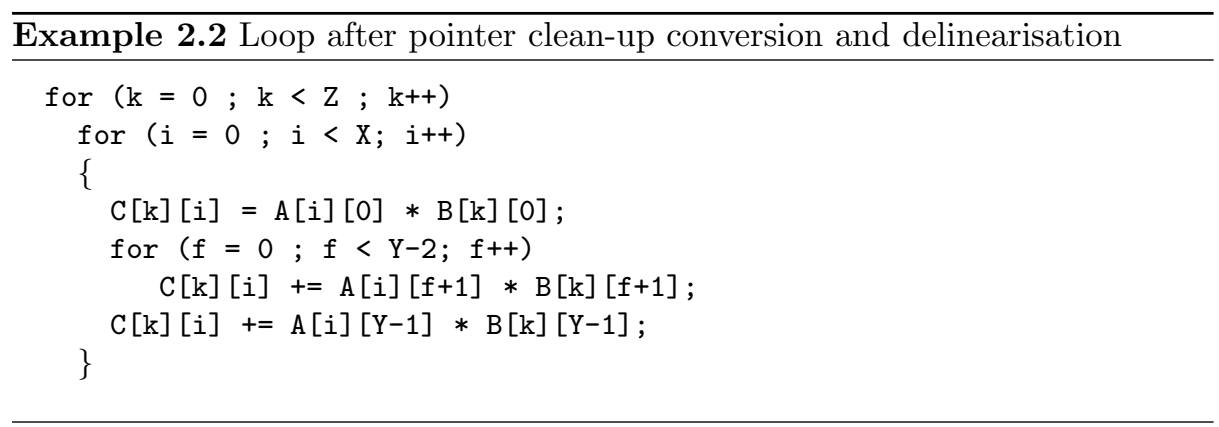

\section{Algorithm}

Pointer conversion is performed in two stages. Firstly, array and pointer manipulation information is gathered. Secondly, this information in used to replace pointer accesses by corresponding explicit array accesses, removing any pointer arithmetic.

\subsection{Assumptions and Restrictions}

The general problems of array dependence analysis and pointer analysis are intractable. After simplifying the problem by introducing certain restrictions, analysis might not only be possible but also efficient.

Pointer conversion may only be applied if the resulting index functions of all array accesses are affine functions. These functions must not depend on other variables apart from enclosing loop induction variables.

To facilitate pointer conversion, guarantee termination and correctness the overall affine requirement can be broken down further into the following restrictions:

1. structured loops

2. no pointer assignments except for initialisation to some array start element

3. no data dependent pointer arithmetic

4. no function calls that might change pointers itself

5. equal number of pointer increments in all branches of conditional statements

Structured loops are loops with a normalised iteration range going from the lower bound 0 to some constant upper bound $N$. The step is normalised to 1 . Structured loop have the Single-Entry/Single-Exit property. 
Pointer assignments apart from initialisations to some start element of the array to be traversed are not permitted. In particular, dynamic memory allocation and deallocation cannot be handled. Initialisations of pointers, however, to array elements may be performed repeatedly and may depend on a loop induction variable. Example 3.1 shows a program fragment with initialisations of the pointers ptr 1 and ptr 2 . Whereas ptr 1 is statically initialised, ptr 2 is initialised repeatedly within a loop construct and with dependence on the outer iteration variable $i$.

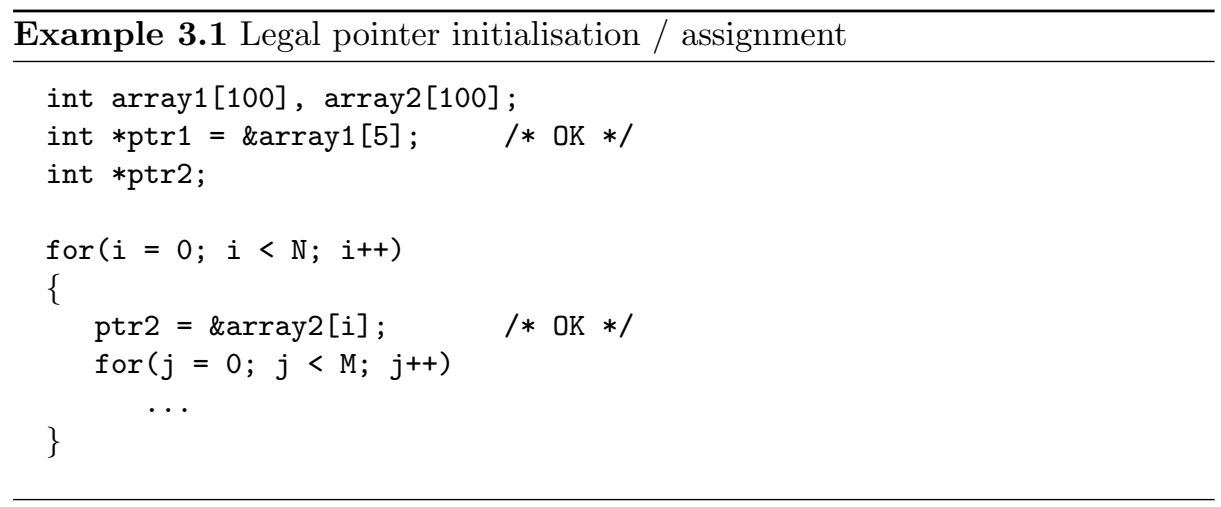

In example 3.2 illegal pointer assignments are shown. A block of memory is dynamically allocated and assigned to ptr1, whereas the initialisation of ptr2 is runtime dependent. The assignment to ptr3 is illegal if the pointer ptrX cannot be statically determined, but acceptable otherwise.

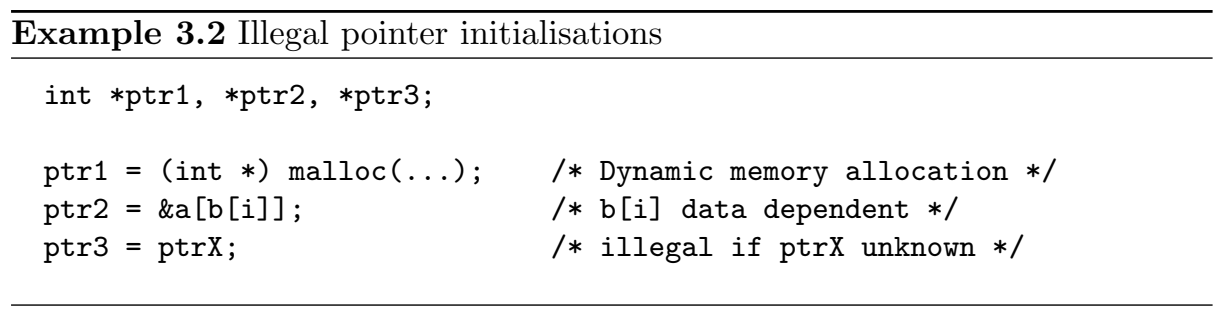

Data dependent pointer arithmetic is the modification of a pointer ( not the value pointed to) in a runtime dependent manner. Although there are powerful methods available e.g. [12] for this problem of pointer or alias analysis, such program constructs are not considered by our algorithm. Pointer expressions that can be statically determined are admissible (see Example 3.3)

Function calls might also modify pointers. Those functions that take pointers to pointers as arguments are disallowed as the actual pointers passed to the 


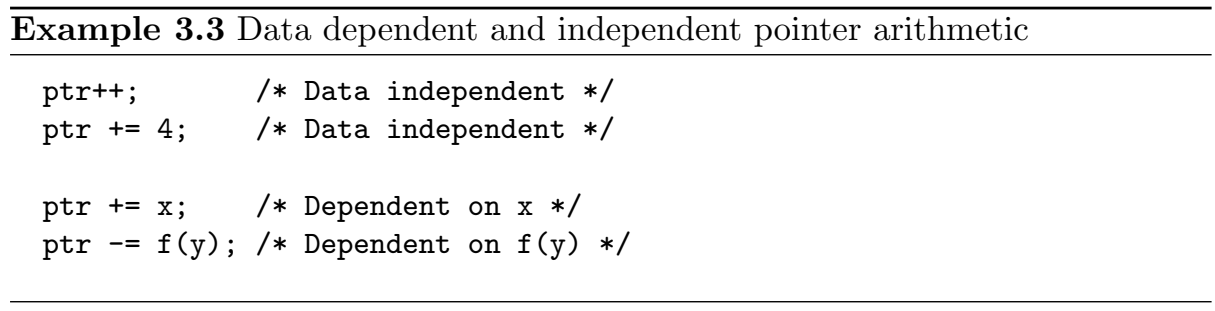

function itself and not only their content can be changed. Example 3.4 illustrates this point. The call to function2 may possibly be handled using inter-procedural pointer analysis [7, but this is beyond the scope of this paper.

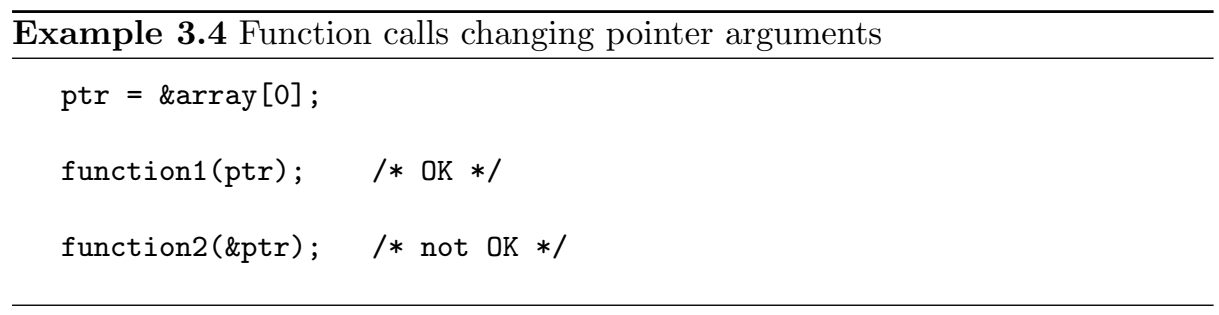

Finally, the number of increments of a pointer must be equal in all branches of conditional statements so that it is possible to statically guarantee that the value of a pointer at any point within the program, is the same regardless of runtime control-flow path. Situations with unequal number of pointer increments are extremely rare and typically not found in DSP code. The first if-statement in example [3.5] is legal, because ptr1 is treated equally is both branches. In the second if-statement the increments are different, therefore this construct cannot be handled.

Note, however, that overlapping arrays and accesses to single arrays via several different pointers are perfectly acceptable. Because the conversion does not require information on such situations, but only performs a transformation of memory access representation, these kind of constructs that often prevent standard program analysis do not interfere with the conversion algorithm.

\subsection{Overall Algorithm}

During data acquisition in the first stage the algorithm traverses the Control Flow Graph (CFG) and collects information regarding when a pointer is given its initial reference to an array element and keeps track of all subsequent changes. Note that only changes of the pointer itself and not of the value of the object pointed to are traced. When loops are encountered, information regarding loop bounds and induction variables is recorded. 


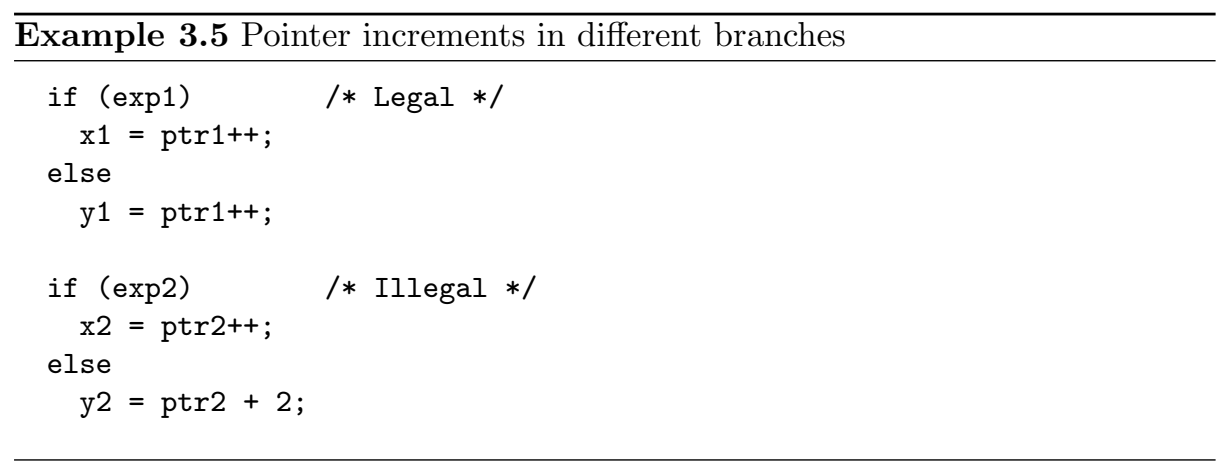

The main objective of the second phase is to replace pointer accesses to array elements by explicit array accesses with affine index functions. The mapping between pointers and arrays can be extracted from information gathered from pointer initialisation in the first phase. Array index functions outside loops are constant, whereas inside loops they are dependent on the loop induction variables. Information on pointer arithmetic collected during the first stage is then used to determine the coefficients of the index functions, Finally pointerbased array references are replaced by semantically equivalent explicit accesses, whereas expressions only serving the purpose of modifying pointers are deleted.

The pointer conversion algorithm can be applied to whole functions and can handle one- and multi-dimensional arrays, general loop nests of structured loops and several consecutive loops with code in between. It is therefore not restricted to handling single loops. Loop bodies can contain conditional control flow.

Algorithm 1 keeps a list of nodes to visit and depending on the type of statement, different actions will be performed. Pointer initialisations and assignments result in updates of the pointer-to-array mapping, whereas loops are handled by a separate procedure. Pointer arithmetic expressions are statically evaluated and pointer-based array references are replaced by equivalent explicit array references. The mapping between pointers contains not only the pointer and corresponding array, but also the initial offset of the pointer within the array and some local offset for keeping track of pointer increments in loop bodies.

The procedure for handling loops is part of the algorithm 1, Similar to the basic algorithm the loop handling procedure performs a pre-order traversal of the nodes of the loop body. Two passes over all nodes are made: The first pass counts the total offset within one loop iteration of pointers traversing arrays, the second pass then is the actual replacement phase. A final stage adjusts the pointer mapping to the situation after the end of the loop.

The algorithm passes every simple node once, and every node enclosed in a loop construct twice. Hence, the algorithm uses time $O(n)$ with $n$ being the number of nodes of the CFG. Space complexity is linearly dependent on the number of different pointers used for accessing array elements, because for every pointer there is a separate entry in the map data structure. 


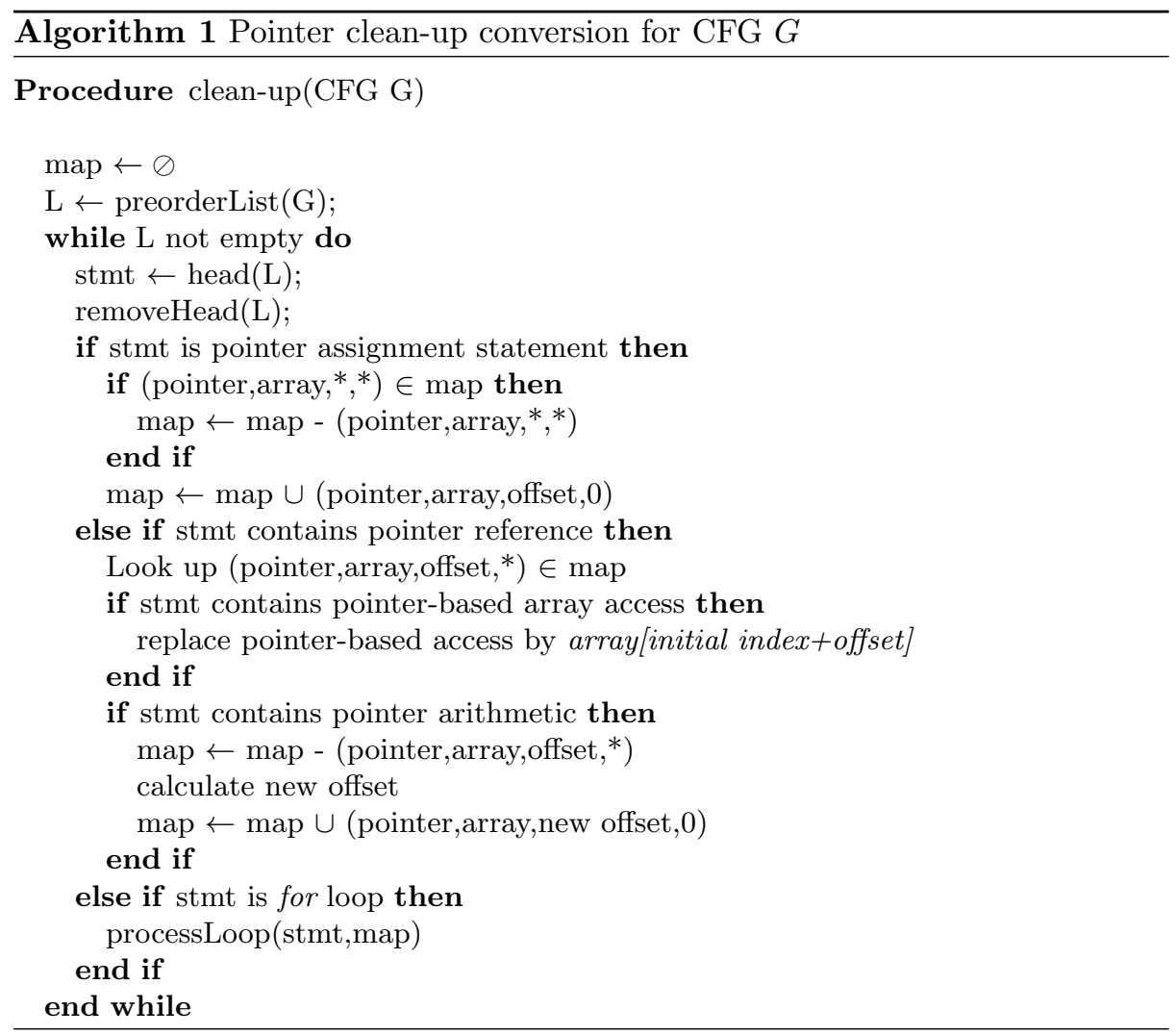

Arrays Passed as Parameters of Functions. If no legal pointer assignment can be found, but the pointer used to traverse an array is a formal parameter of the function to be processed, it can be used as the name of the array [4].

Table 1. Absolute times for biquad_N_sections and the TriMedia

\begin{tabular}{|l|c|c|c|c|c|}
\hline Benchmark & Level & \multicolumn{2}{|c|}{ TriMedia } & \multicolumn{2}{c|}{ Pentium II } \\
\hline \multirow{3}{*}{ biquad } & & Array & Pointer & Array & Pointer \\
& -O0 & 11 & 9 & 1130 & 980 \\
& -O1 & 11 & 8 & 490 & 360 \\
& -O2 & 8 & 8 & 290 & 360 \\
& -O3 & 9 & 8 & 360 & 350 \\
\hline
\end{tabular}

Loop Nests. Perfect loop nests of structured loops are different from simple loops in the way that the effect of a pointer increment/decrement in the loop body not only multiplies by the iteration range of its immediately enclosing loop 
Procedure processLoop(statement stmt, mapping map)

\{count pointer increments in loop body and update map\}

$\mathrm{L}=$ preorderList(loopBody)

while L not empty do

stmt $\leftarrow \operatorname{head}(\mathrm{L})$;

removeHead $(\mathrm{L})$;

if stmt contains array arithmetic then

Update increment in (pointer,array,offset,increment)

end if

end while

\{replace pointer increments according to map

$\mathrm{L}=$ preorderList(loopBody)

while L not empty do

if stmt contains pointer reference then

Look up (pointer,array,offset,increment) $\in$ map

Look up (pointer,local offset) $\in$ offsetMap

if (pointer,local offset) $\notin$ offsetMap then offsetMap $\leftarrow$ offsetMap $\cup$ (pointer, 0$)$

end if

if stmt contains pointer-based array access then

index $\leftarrow$ increment $\times$ ind.var. + offset + local offset

replace pointer-based access by array[index]

end if

if stmt contains pointer arithmetic then

Update local offset in map

end if

end if

end while

\{adjust all mappings to situation after end of loop\}

for all (pointer, $\left.{ }^{*},{ }^{*},{ }^{*}\right) \in \operatorname{map}$ do

update offsets in map

end for

construct, but by the ranges of all outer loops. Therefore, all outer loops have to be considered when converting pointers of a perfect loop nest.

Handling perfect loop nests does not require extra passes over the loop. It is sufficient to detect perfect loop nest and descend to the inner loop body while keeping track of the outer loops. Once the actual loop body is reached conversion can be performed as usual, but with incorporating the additional outer loop variables and loop ranges as part of the index functions. Hence, asymptotical run-time complexity of the algorithm is not affected.

General loop nests can similarly be handled with a slightly extended version of the basic algorithm, by tracking which loop a pointer is dependent upon. The introductory example 1.1 illustrates a general loop nest and its conversion. 


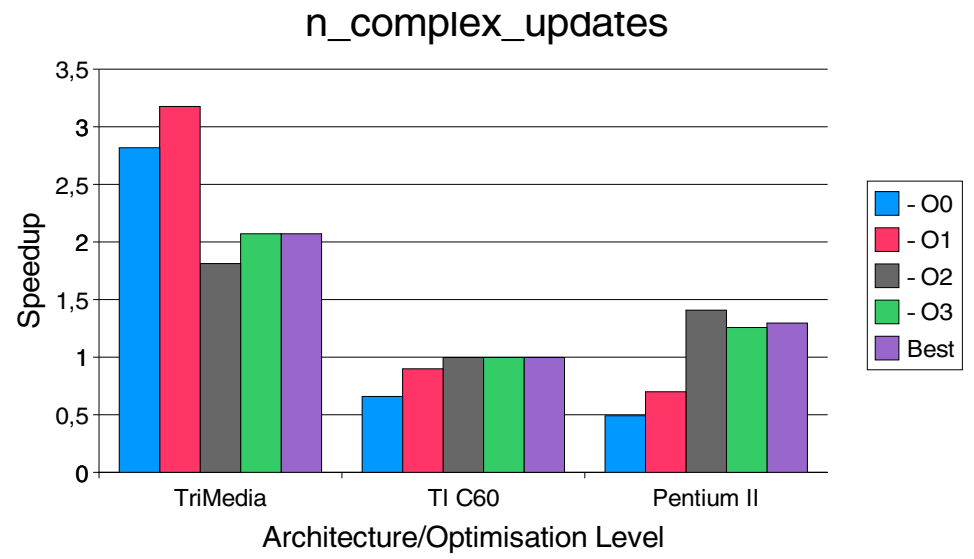

Fig. 1. Performance comparison of $n_{-}$complex_updates benchmark

\section{Experiments}

The pointer clean-up conversion algorithm has been implemented as a prototype and integrated into the experimental source to source Octave compiler. After the application of the transformation on source-to-source level the resulting code is then used as an input for the C compilers of the Philips TriMedia TM-1 (compiler version 5.3.4), the Texas Instruments TMS320C6x (compiler version 1.10) and the Intel Pentium II (Linux 2.2.16, gcc version 2.95.2). Performance data was collected by executing the programs on two simulators (TM-1, TI C60) and a real machine (PII), respectively.

In order to quantify the benefit of applying the pointer clean-up conversion to DSP applications, program execution times for some programs of the DSPstone benchmark suite were determined. The speedups of the programs with explicit array accesses with respect to the pointer-based versions for the same optimisation level were calculated. As a DSP application developer typically wants the best performance we determined the optimisation level that gave the best execution time for the pointer and array codes separately. Due to the complex interaction between compiler and architecture, the highest optimisation level does not necessarily give the best performance. Thus the ratio between the best pointer-based version and the best explicit array access version is also presented.

Table 1 shows an example of the absolute performance figures for the biquad_N_sections benchmark on the TriMedia and Pentium II architectures that were the used for the computations of the speedup. Due to different optimi- 
sations performed by the compilers at each optimisation level and different units of execution time, the reported times cannot be compared directly. Therefore, relative speedups based on the pointer-based program at the same optimisation level are shown in the following diagrams. As stated above, the minimal execution times are not necessarily achieved with the highest available optimisation level. Thus, it is reasonable to give an additional speedup measure when comparing the best pointer-based and explicit array based versions. For example, the shortest execution time achieved on the Pentium II was 350 for the pointerbased version and 290 for the explicit array access based version. Hence, the best speedup is $\frac{350}{290}=1.21$. The figures [1 to 6 visualise the speedup measures for all three architectures and all optimisation levels.

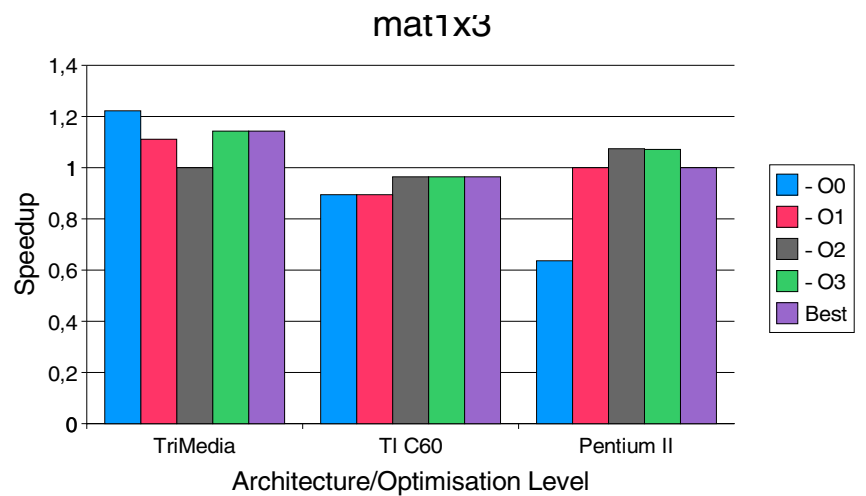

Fig. 2. Performance comparison of mat1x3 benchmark

\subsection{TriMedia}

The performance of the TriMedia compiler/architecture combination usually benefits from using explicit array accesses rather than pointer-based array accesses. When there are no or only a few simple further optimisations applied (optimisation levels $\mathrm{O} 0$ and $\mathrm{O} 1$ ), the program versions with explicit array accesses are significantly faster than the pointer-based programs. The difference can be up to $218 \%$ (n_complex_updates). For higher optimisation levels the differences become smaller. Although some of the original programs take less time than the transformed versions, in many cases there can be still some performance gain expected from pointer clean-up conversion. The pointer-based programs tend to 
be superior at optimisations level O2, but the situation changes again for level O3 and the "best" case. This "best" case is of special interest since it compares the best performance of a pointer-based program to that of an explicit array access based program. The best explicit array based programs perform usually better or at least as well as the best pointer-based programs, only in one case (dot_product) a decrease in performance could be observed.

In figure 1 the speedup results for the $\mathrm{n}_{-}$complex_updates benchmark are shown. This rather more complex benchmark program shows the largest achieved speedups among all tests. The speedup reaches its maximum 3.18 at the optimisation level O1, and is still at 2.07 when comparing the best versions. This program contains a series of memory accesses that can be successfully analysed and optimised in the explicit array based version, but are not that easily amendable to simple pointer analyses. Although such large speedups are not common for all evaluated programs, it shows clearly the potential benefit of the pointer clean-up conversion.

Figure 2 compares the performances of the mat1x3 benchmark . All speedups for the TriMedia architecture are $\geq 1$. The achieved speedups are more typical for the set of test programs. An increase of $14 \%$ in performance can be observed for the best explicit array based program version. With only a few other optimisations enabled the performance benefit of the transformed formed program is even higher.

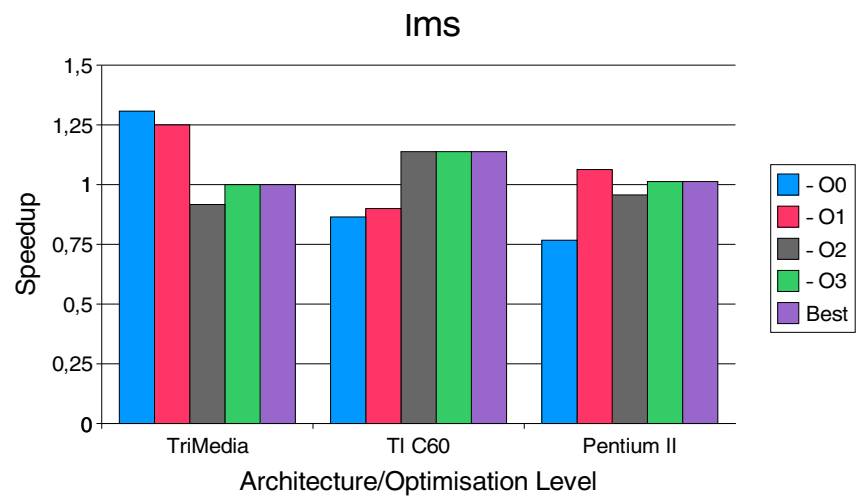

Fig. 3. Performance comparison of lms benchmark

The figures 3 and 4 represent the results of the lms and matrix 1 benchmark, respectively. As before, a significant speedup can be achieved at optimisation 
levels $\mathrm{O} 0$ and $\mathrm{O} 1$. The performances of the explicit array accesses based versions are actually below those of the pointer-based versions at level O2, but at the highest level the transformed programs perform better or as well as the original versions. Although there is only a small speed up observable in these examples, it shows that the use of explicit array accesses does not cause any run-time penalty over pointer-based array traversals, but still provide a representation that is better suitable for array data dependence analysis.

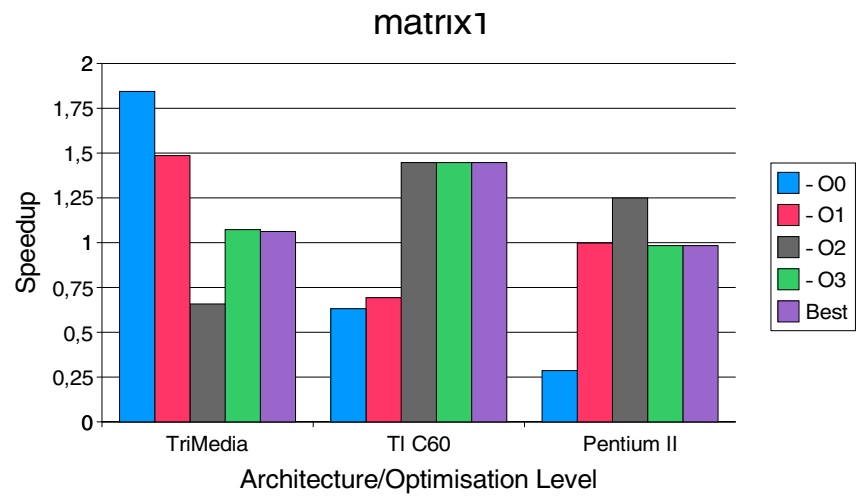

Fig. 4. Performance comparison of matrix1 benchmark

In general, with pointer clean-up conversion some substantial benefit can be achieved without the need for further optimisations. On higher optimisation levels the transformed programs still perform better or at least as good as the pointer-based programs. Because all evaluated programs are not too complex, the original programs can often perform as good as the transformed programs at higher levels. But as shown in figure1 for more complex programs the conversion provides some substantial advantage.

\subsection{Pentium II}

The Pentium II is a general-purpose processor with a super-scalar architecture. Additionally, it supports a CISC instruction set. This makes the Pentium II quite different from the TriMedia. However, many signal processing applications are run on Desktop PCs in which the Intel Processor is commonly found. Therefore, this processor is included in this evaluation.

The combination of the Intel Pentium II and the gcc compiler produces results that differ significantly from that of the TriMedia. The performance of 
the program versions after pointer conversion is quite poor without any further optimisations, but as the optimisation level is increased the transformed programs often outperform the original versions. The maximum speedup of the transformed programs is usually achieved at optimisation levels O2 and O3, respectively.

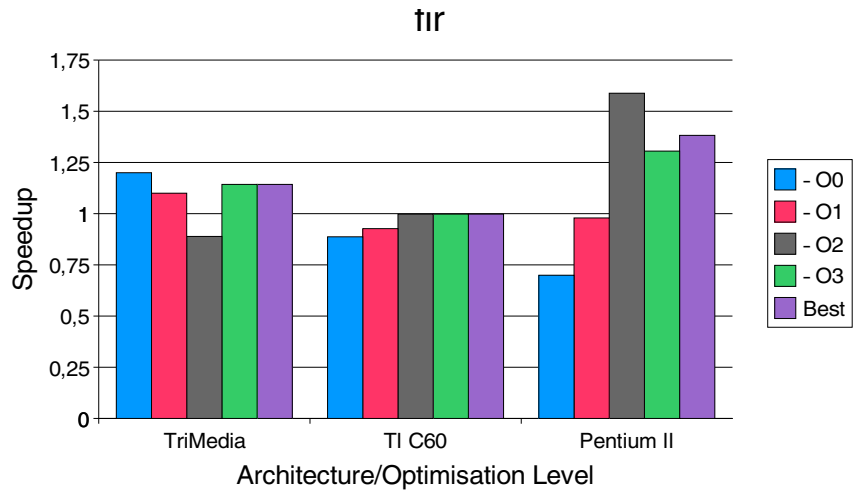

Fig. 5. Performance comparison of fir benchmark

In figure 5 the results for the $\mathrm{f}$ ir benchmark are charted. Initially, the pointer transformation has negative effect on the performance of this program. A significant speedup can be observed for the higher optimisation levels. The maximum speedup achieved at level $\mathrm{O} 2$ is 1.59 and when comparing the two best versions each, the speedup of 1.38 can still be reached. The results of the fir $2 \mathrm{dim}$ benchmark are represented in figure [6 As before the performance of the transformed version is inferior at optimisation levels $\mathrm{O} 0$ and $\mathrm{O} 1$, but it increases at $\mathrm{O} 2$ and O3. The performance can be increased by up to $6 \%$ in this case.

In general, the achievable speedups on the Pentium II architecture are not as large as they are on the TriMedia. It is not yet clear whether this is a consequence of architectural properties or the influence of the different compilers. However, an increase in the performance can be observed for the Intel processor, in particular at the higher optimisation levels.

\subsection{TI TMS320C6x}

The overall performance improvement of the Texas Instruments C60 lies between that of the Pentium II and the TM-1. In two cases the pointer conversion has no 


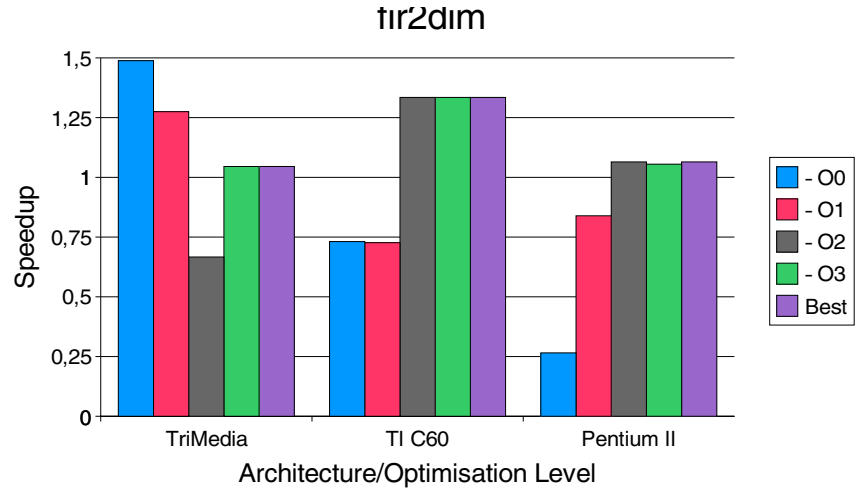

Fig. 6. Performance comparison of fir2dim benchmark

effect on the execution time of the programs, in one case there is actually a small degradation in performance, mat $1 x 3$, and in three cases there is a variability in terms of relative performance improvement with respect to the optimisation level. Increasing the optimisation level for the C60 improves the impact of the pointer conversion.

If we compare the behaviour of the two processors intended for embedded systems, we see that there is little correlation in their behaviour with respect to the conversion algorithm. The case where the TM-1 gains most from our technique, n_complex_updates has no impact on the C60; conversely the case where the C60 most benefits, matrix1, has little impact on the TM-1. From this we can conclude that the optimisation behaviour of both compilers is opaque and that further analysis is required to explain the behaviour in order to drive further optimisations.

\section{Related Work}

Previous work on transforming pointer accesses into a more suitable representation has focused on simplifying pointer expressions rather than transforming them into explicit array references. For example, Allan and Johnson [1] use their vectorisation and parallelisation framework based on $\mathrm{C}$ as an intermediate language for induction variable substitution. Pointer-based array accesses together with pointer arithmetic in loops are regarded as induction variables that can be converted into expressions directly dependent on the loop induction variable. The generated pointer expressions are more amendable to vectorisation than the 
original representation, but this approach still does not fully regenerate the index expressions. For the main objective of vectorisation the method only treats loops individually rather than in a context of a whole function. The approach is based on a heuristic that mostly works efficiently, although backtracking is possible. Hence, in the worst case this solution is inefficient. No information is supplied about treatment of loop nests and multi-dimensional arrays. In his case study of the Intel Reference Compilers for the i386 Architecture Family Muchnick 9] mentions briefly some technique for regenerating array indexes from pointer-based array traversal. No more details including assumptions, restrictions or capabilities are given. The complementary conversion, i.e. from explicit array accesses to pointer-based accesses with simultaneous generation of optimal AGU code, has been studied by Leupers [5] and Araujo [2].

\section{Conclusion}

The contribution of this paper has been to introduce a new technique for transforming $\mathrm{C}$ code with pointer-based array accesses into explicit array accesses to support existing array data flow analyses and optimisations on DSP architectures. The approach has been implemented and integrated into the experimental Octave compiler and tested on examples of the DSPstone benchmark suite. Results show a significant $11.95 \%$ reduction in execution time. The generation of efficient address generation code is improved since modern compilers are able to analyse array accesses and to generate optimised code for memory accesses that does not rely on the use of pointers at the source level.

We believe the use of explicit array accesses to be the key to automatic parallelisation of DSP applications for Multiprocessor DSP architectures. Future work will focus on distributing computation and data on different co-operating DSPs while making the best use of ILP and coarse-grain parallelism.

\section{References}

1. Allen R. and Johnson S., Compiling C for Vectorization, Parallelization, and Inline Expansion, Proceedings of the SIGPLAN '88 Conference of Programming Languages Design and Implementation, pp. 241-249, Atlanta, Georgia, June 2224, 1988

2. de Araujo, Guido C.S., Code Generation Algorithms for Digital Signal Processors, Dissertation, Princeton University, Department of Electrical Engineering, June 1997.

3. Duesterwald E., Gupta R.and Soffa M., A Practical Data Flow Framework for Array Reference Analysis and its Use in Optimizations, Proceedings of the SIGPLAN Conference on Programming Languages Design and Implementation, 28(6), pp. 67-77, Albuquerque, New Mexico, 1993.

4. Kernighan, Brian W. and Ritchie, The C Programming Language, Second Edition, Prentice Hall, Englewood Cliffs, New Jersey, 1988.

5. Leupers R., Novel Code Optimzation Techniques for DSPs, 2nd European DSP Education and Research Conference, Paris, France, 1998. 
6. Liem C., Paulin P., Jerraya A., Address Calculation for Retargetable Compilation and Exploration of Instruction-Set Architectures Proceedings of the 33rd Design Automation Conference, Las Vegas, Nevada 1996.

7. Lu, J., Interprocedural Pointer Analysis for C, Ph.D. thesis, Department of Computer Science, Rice University, Houston, Texas, 1998. SASIMI, Osaka, 1997.

8. Maydan, Dror.E., John L. Hennessy, and Monica S. Lam., Effectiveness of Data Dependence Analysis, International Journal of Parallel Programming, 23(1):6381, 1995.

9. Muchnick, Steven.S., Advanced Compiler Design and Implementation, Morgan Kaufmann Publishers, San Francisco, California, 1997.

10. Numerix-DSP Digital Signal Processing Web Site, http://www.numerix-dsp.com/c_coding.pdf, 2000.

11. O'Boyle M.F.P and Knijnenberg P.M.W., Integrating Loop and Data Transformat ions for Global Optimisation, PACT '98, Parallel Architectures and Compiler Technology, IEEE Press, October 1998.

12. Wilson, R.P., Efficient Context-Sensitive Pointer Analysis for C Programs, Ph.D. thesis, Stanford University, Computer Systems Laboratory, December 1997.

13. Zivojnovic, V., Velarde J.M., Schlager C. and Meyr H., DSPstone: A DSPOriented Benchmarking Methodology, Proceedings of Signal Processing Applications \& Technology, Dallas 1994 .

14. Zima H., Supercompilers for Parallel and Vector Computers, ACM Press, 1991. 